\title{
A new look into the Helios dust experiment data: presence of interstellar dust inside the Earth's orbit
}

\author{
N. Altobelli ${ }^{1}$, E. Grün ${ }^{12}$, and M. Landgraf ${ }^{3}$ \\ 1 MPI für Kernphysik, Saupfercheckweg 1, 69117 Heidelberg, Germany \\ e-mail: nicolas.altobelli@jpl.nasa.gov \\ 2 HIGP, University of Hawaii, Honolulu, USA \\ e-mail: eberhard.gruen@mpi-hd.mpg.de \\ 3 ESA/ESOC, Germany \\ e-mail: markus. landgraf@esa.int
}

Received 25 July 2005 / Accepted 21 October 2005

ABSTRACT

\begin{abstract}
An analysis of the Helios in situ dust data for interstellar dust (ISD) is presented in this work. Recent in situ dust measurements with impact ionization detectors on-board various spacecraft (Ulysses, Galileo, and Cassini) showed the deep penetration of an ISD stream into the Solar System. The Helios dust data provide a unique opportunity to monitor and study the ISD stream alteration at very close heliocentric distances. This work completes therefore the comprehensive picture of the ISD stream properties within the heliosphere. In particular, we show that gravitation focusing facilitates the detection of big ISD grains (micrometer-size), while radiation pressure prevents smaller grains from penetrating into the innermost regions of the Solar System. A flux value of about $2.6 \pm 0.3 \times 10^{-6} \mathrm{~m}^{-2} \mathrm{~s}^{-1}$ is derived for micrometer-size grains. A mean radiation pressure-to-gravitation ratio (so-called $\beta$ ratio) value of 0.4 is derived for the grains, assuming spheres of astronomical silicates to modelize the grains surface optical properties. From the ISD flux measured on the Helios trajectory, we infer a lower limit of $3 \pm 3 \times 10^{-25} \mathrm{~kg} \mathrm{~m}^{-3}$ to the spatial mass density of micron-sized grains in the Local Interstellar Cloud (LIC). In addition, compositional clues for ISD grains are obtained from the data provided by the time-of-flight mass spectrometer subsystem of the Helios instrument. No clustering of single minerals is observed but rather a varying mixture of various minerals and carbonaceous compounds.
\end{abstract}

Key words. ISM: dust, extinction - interplanetary medium - meteors, meteoroids

\section{Introduction}

Interstellar dust (ISD) is a key component of the interstellar medium (ISM). ISD grains ensure an efficient transport of heavy elements through the galaxy and are closely related to its chemical evolution. Astronomical observations of star light extinction and reddening succeeded in providing meaningful ISD physical parameters. Compositional models were inferred (Draine \& Lee 1984; Li \& Greenberg 1997) together with a standard ISD size distribution in the ISM, the famous "MRN distribution" (Mathis et al. 1977). ISD grains located in the closest neighborhood of the Solar System, the local interstellar cloud (LIC), are of strong interest as typical grains present in the diffuse ISM. Unfortunately, optical observations fail to provide insights on ISD grains in the LIC, owing to the very small optical depth of this region (Frisch et al. 1998).

In situ measurements performed by dust instruments onboard spacecraft have turned out to be an outstanding method for retrieving physical properties of individual ISD grains. One of the best suited detection methods, using impact ionization, was developed over the last 30 years. This method allows studying the mass, impact velocity, elemental composition and charge of sub-micrometer to micrometer size particles. For a review of the successive generations of dust impact ionization detectors, we refer the reader to Grün (1981); Grün et al. (1992a,b); Srama et al. (2003). Milestones in ISD in situ detection were achieved by the dust instrument on-board the Ulysses and Galileo spacecraft. It was shown that a collimated ISD stream passes through the Solar System because of the Sun's motion relative to the LIC. The derived heliocentric velocity of the ISD flux is around $26 \mathrm{~km} \mathrm{~s}^{-1}$ (Grün et al. 1994) and the downstream dust flow direction is compatible with the direction of the interstellar helium flux at about $75^{\circ}$ and $-5^{\circ}$ ecliptic latitude (Witte et al. 1993; Baguhl et al. 1995). The bulk of the mass distribution is located around $10^{-16} \mathrm{~kg}$ (Landgraf et al. 2000), implying a grain radius of about $0.4 \mu \mathrm{m}$ if one assumes a material bulk density of $2500 \mathrm{~kg} \mathrm{~m}^{-3}$ (Burns et al. 1979). Therefore, the dominant contribution to the Ulysses data sample consists surprisingly of grains larger than the "classical" ones described by the MRN distribution.

The dynamics of individual ISD grains are ruled by three perturbating accelerations: the Sun's gravitation, solar radiation pressure, and the Lorentz acceleration resulting from the 


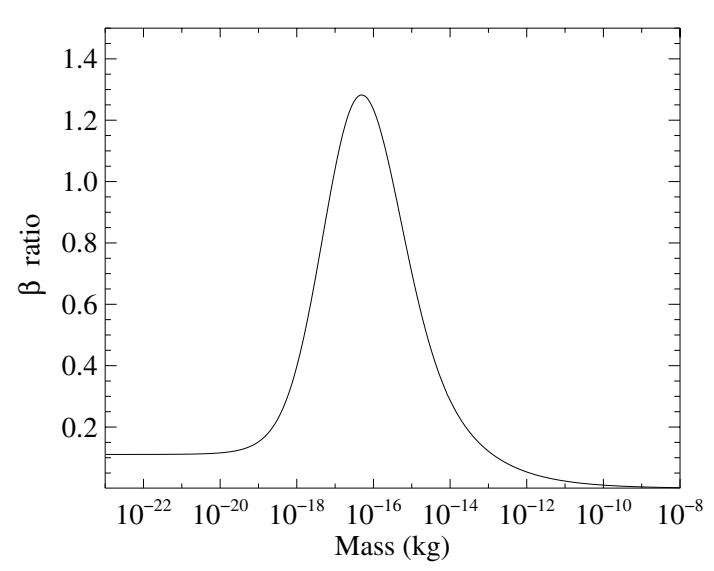

Fig. 1. Ratio of radiation pressure to gravitational force $\beta$, calculated as function of the particle mass. The shape of the particle is assumed to be spherical and homogeneous. The optical properties are those calculated for astronomical silicates. The results presented here, calculated with the Mie theory, are taken from Gustafson (1994).

coupling of charged grains with the interplanetary magnetic field (IMF) (Kimura \& Mann 1998). IMF coupling is most efficient for small grains $(<0.2 \mu \mathrm{m})$, owing to their higher chargeto-mass ratio. As a result, small grains are deflected away from the Sun and cannot reach the innermost region of the Solar System (Grün et al. 1994; Landgraf et al. 2003). In contrast, the dynamics of bigger grains is ruled dominantly by radiation pressure and gravitation, whose relative strengths are related through their so-called $\beta$ ratio. Assuming a spherical shape, modeling the dust grains optical properties provides theoretical values for $\beta$ (Gustafson 1994). Figure 1 shows that the radiation pressure can, for certain grain mass values, overcome gravitation $(\beta>1)$. This results in zones of avoidance within the heliosphere, identified in situ, inside which ISD grains within a given mass range cannot penetrate (Landgraf et al. 2000; Mann \& Grün 1996). As a whole, the heliospheric interactions separate the ISD grains according to their mass and weaken the ISD flux the closer one gets to the Sun.

However, a still significant ISD stream has been observed around $1 \mathrm{AU}$ with dust instruments carried by the Cassini and Galileo spacecraft (Altobelli et al. 2003, 2004). This finding pointed out the need for an analysis for ISD from dust datasets obtained at very close heliocentric distances (less than $1 \mathrm{AU}$ ), to complete the picture of the ISD stream dynamics within the Solar System. This opportunity was offered by the Helios mission. An analysis for ISD of the Helios dust data had never been performed until now, mainly because at the time of the first data analysis the presence of ISD within the Solar System was more a theoretical suggestion (Levy \& Jokipii 1976; Morfill \& Grün 1979; Gustafson \& Misconi 1979) than a proved experimental fact. In this work, we reanalyze the Helios dust data for ISD in the light of the recent knowledge on the ISD stream interactions with the heliosphere, based on the Cassini, Ulysses, and Galileo dust data. After a brief description of the mission and of the dust instrument in Sect. 2, the data are presented in Sect. 3. The identification of ISD grains follows in Sect. 4 . The results, including compositional information, are discussed in Sect. 5.

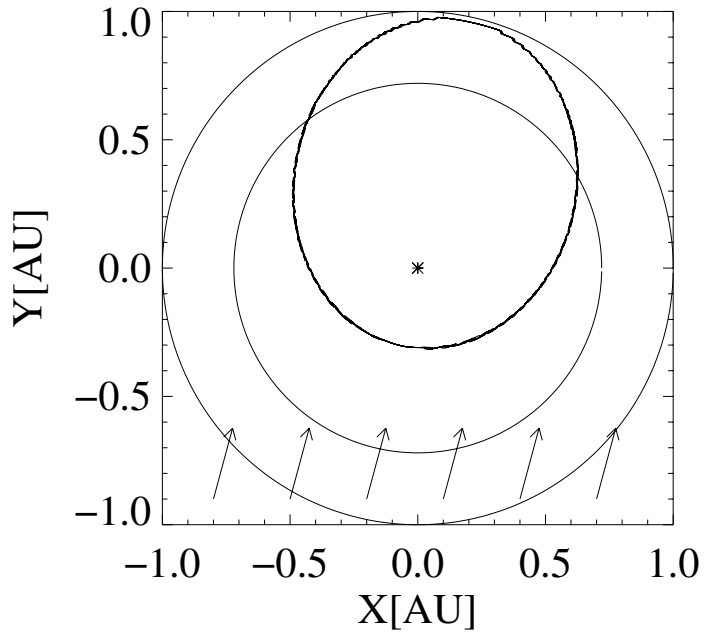

Fig. 2. Trajectory of the Helios spacecraft in the ecliptic reference frame. The perihelion of the elliptic orbit is located about $0.3 \mathrm{AU}$ from the Sun, and the aphelion at Earth's orbit distance from the Sun. The orbits of the Earth and Venus are also plotted. The arrows represent the injection direction of the ISD stream into the heliosphere. Note that the ISD stream direction is almost parallel to the apsides line of the Helios trajectory.

\section{Mission description and instrumentation}

The Helios spacecraft was the result of a US-German cooperation. Launched in 1974, the goal of the mission was to reach an orbital perihelion at $0.3 \mathrm{AU}$ from the Sun (see Fig. 2), performing measurements of the IMF, the solar wind, cosmic radiations, the zodiacal light, and the interplanetary dust distribution. Three months after the launch, the preliminary mission was successfully complete, and an extended mission was begun, consisting of an additional 5 years measurements on the same orbit. The Helios trajectory lies in the ecliptic plane. The eccentricity of the elliptical orbit was about $e=0.56$, the perihelion was located at $0.31 \mathrm{AU}$ from the Sun, and the aphelion at $0.98 \mathrm{AU}$. The argument of the perihelion was $258.4^{\circ}$. The orbital period was about 190 days. The spacecraft was spinning around its antenna axis (perpendicular to the ecliptic plane), the rotation being parameterized by the azimuth angle $\theta$. The origin of the $\theta$ angle is chosen such that for both dust instruments $\theta=90^{\circ}$ indicates the Sun direction and $\theta=0^{\circ}$ gives the perpendicular direction towards the spacecraft apex motion.

The Helios dust instrument's physical mechanism is based on the impact ionization generated upon impact of a highvelocity projectile onto a solid target (Dietzel et al. 1973). Both the impact velocity and the mass of the impactor can be derived. In addition, the Helios instrument had the first timeof-flight mass spectrometer subsystem ever flown in space, providing chemical elemental composition information about the impactor. The target is a venetian blind consisting of gold strips held at ground potential. The constituents of the impact plasma are electrons, positive and negative ions, neutral atoms or molecules, and residual fragments of the impactor and target. Electrostatic fields are used to separate the positive charges from the negative ones generated upon the impact. The mass of the particles can be derived from both using the rise time and 

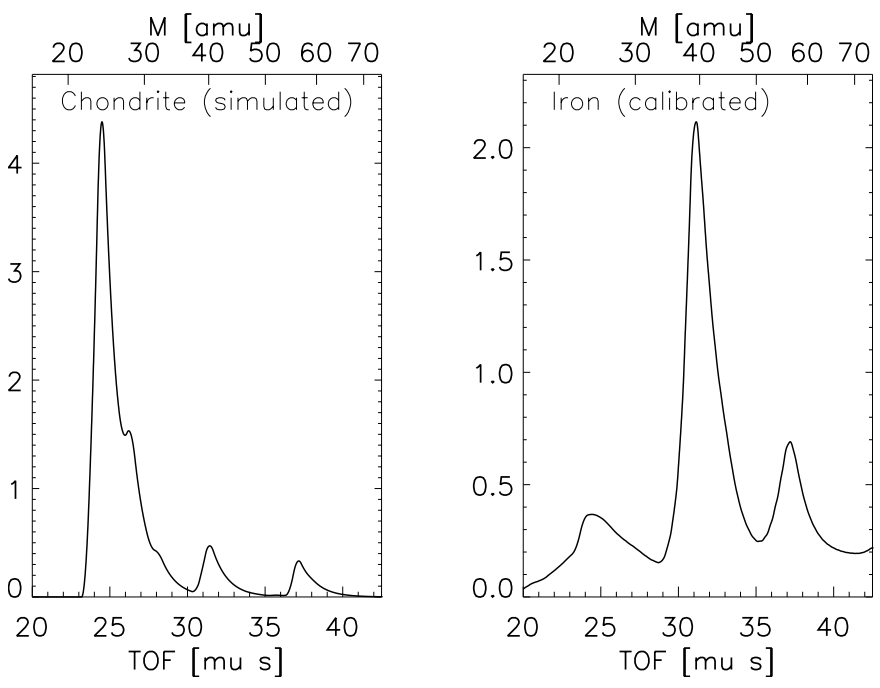

Fig. 3. Examples of typical Helios spectra. Left: simulated spectrum (type $\mathrm{Ch}$ ) derived from the composition of chondritic micrometeorites (Brownlee 1978), ion yield values taken from (Sparrow 1977), and taking into account of the line width of the Helios instruments. Right: spectrum (type $\mathrm{Fe}$ ) obtained from an impact of an iron projectile during laboratory calibration experiments (Grün 1981). Both spectra have been normalized to constant area.

the amplitude of the charge signals (Eichhorn 1978a,b; Grün et al. 1992a,b). The calibration equation for the Helios instrument, as provided in Grün (1981) is

$Q=K \cdot m^{\alpha} v^{\beta}$

with $K=4.07 \times 10^{-5}, \alpha=1$ and $\beta=2.7$. There is proportionality between the impactor mass and the impact charge.

Behind the target is a negatively biased grid (g1) that draws the ions generated upon impact into the interior of the sensor. After most ions have entered the sensor, a further grid (g2) is switched to high negative voltage. Ions between the two grids $\mathrm{g} 1$ and $\mathrm{g} 2$ are accelerated and reach the ion detector (multiplier) after $80 \mathrm{~cm}$. Because of this time-lag focusing (Wiley \& McLaren 1955), a definite start time of mass spectrum and a mass resolution $\frac{M}{\Delta M}$ between 5 and 10 is reached. This focusing has been optimized for masses between 16 to 75 a.m.u (atomic mass unit). Ions of smaller or bigger masses are lost or contribute to the background current at the ion collector. Time-of-flight (TOF) mass spectra are recorded from 20 to 42.5 microseconds after the voltage switch which corresponds to masses 16 to 75 a.m.u.

The instrument has been calibrated with projectiles consisting of iron, quartz, soda lime glass, aluminum, aluminum oxide, Kaolin, and Polystyrene at the dust accelerators at Max Planck Institut for Nuclear Physics, Heidelberg, and at NASA Ames Research Center, Moffett Field (Dalmann et al. 1977). Figure 3 shows two examples of typical spectra. The iron type $\mathrm{Fe}$ account for iron rich particles like those obtained during laboratory experiments, while the chondritic type Ch shows features that are typical for silicate-rich particles (Grün 1981). The Ch spectrum has been calculated theoretically assuming a composition of chondritic micrometeorites (Brownlee 1978) and ion yield values taken from Sparrow (1977). This spectrum

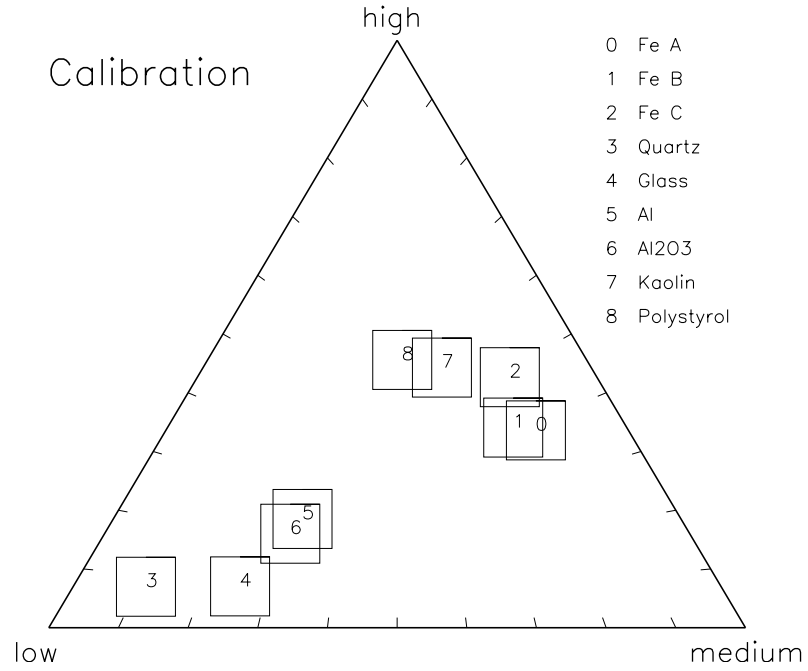

Fig. 4. Three-phase diagram of calibration spectra. The mass range from 16 to 75 a.m.u is separated into three intervals: low masses: 16 to 35 a.m.u, medium masses: 35 to 50 a.m.u, and high masses: 50 to 74 a.m.u. The ion abundance of calibration spectra in these mass intervals is displayed. Along the triangle axes the ion abundance varies from 0 to 1 . The Helios instrument has been calibrated with projectiles consisting of iron (at three different speed regimes: Fe A $2.2 \mathrm{~km} \mathrm{~s}^{-1}$, FE B $5 \mathrm{~km} \mathrm{~s}^{-1}$, and Fe C $7 \mathrm{~km} \mathrm{~s}^{-1}$ ), quartz, soda lime glass, aluminium, aluminium oxid, Kaolin, and Polystyrene (Dalmann et al. 1977). The squares mark the mean positions and extent of mass spectra measured during calibration.

is similar to the glass and quartz spectra observed in the calibrations. Because of the low mass resolution of the spectra, the mass range is separated into three regions: low masses: 16 to 35 a.m.u, medium masses: 35 to 50 a.m.u, and high masses: 50 to 74 a.m.u. All calibration spectra are presented in a three phases diagram (see Fig. 4). Quartz, glass, aluminium compounds and similarly chondritic IDPs of type $\mathrm{Ch}$ would be located in the low ion mass region. Spectra of iron particles of various impact speeds have the lowest content of ions in the low mass region. The highest content of high mass (molecular) ions (>70 a.m.u) had the carbonaceous Polystyrene and the weathered mineral Kaolin. Although these TOF mass spectra give only a rough elemental composition of the detected dust particle, their resolution was high enough to show evidence of several different types of spectra.

The instrument showed a non-nominal behavior for higher energetic impacts due to saturation of one or both of the impact charge channels. For a given particle mass, the higher the impact velocity, the bigger is the amount of impact charge produced (see Eq. (1)), and the bigger the measurement bias that can occur. It has been found experimentally that the ion collection efficiency is only $1 \%$ to $10 \%$ of the electron collection efficiency (Grün 1981). Furthermore, the ratio of the ion charge yield $Q I$ to the electron charge yield $Q E$ appears to be a function of $v$ as shown below:

$\frac{Q I}{Q E} \sim v^{\gamma}$,

with $\gamma=-1.7$ for the Helios instrument (Grün 1981). Thus, for fast and big grains, the electron channel is more likely to 


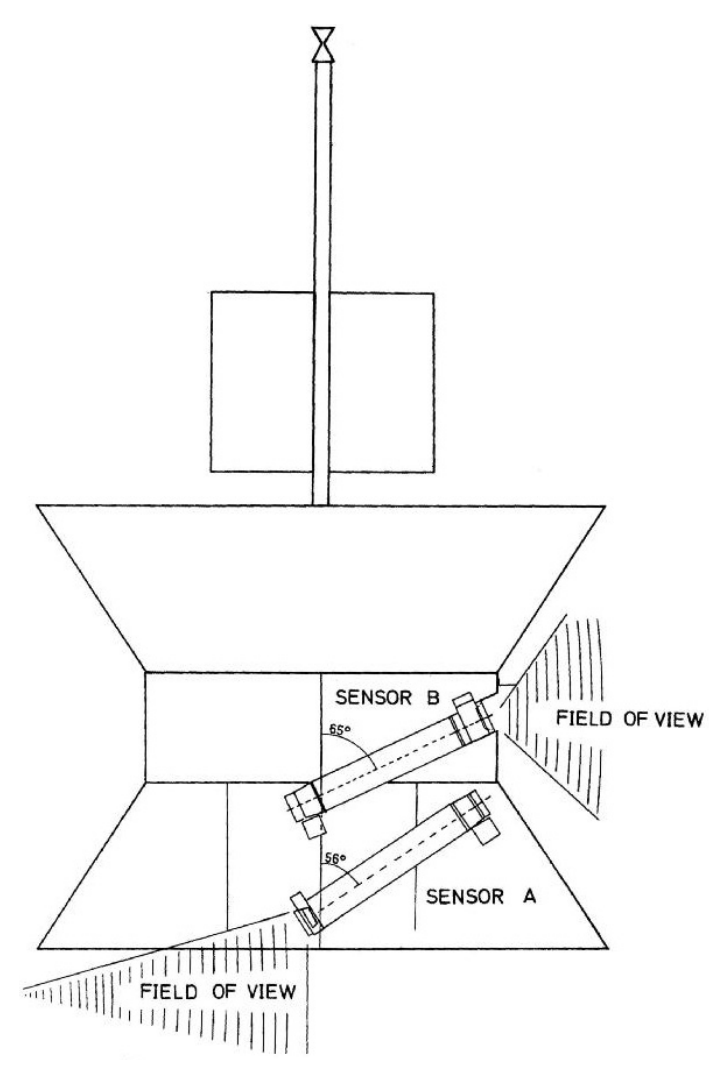

Fig. 5. Schematic of the Helios spacecraft, carrying the twin dust sensors. Sensor B is also called the ecliptic sensor, since it is sensitive to dust particles on low inclined orbits, while Sensor A is called the south sensor, since it is sensitive to particles coming from the ecliptic south direction.

saturate than the ion channel and may introduce an underestimation of the mass measurement. Furthermore, ongoing laboratory work suggest that the collection efficiency of electrons released during high energetic impacts becomes problematic (F. Mueller, private communication). The analysis of the ISD dust component in the Cassini and Galileo data also support the idea that only the ion channel should be considered as reliable in case of high energetic impactors (Altobelli et al. 2003, 2004). For that reason, we based the work reported here on the measurements of $Q I$ only.

Two twin instruments were mounted on the Helios spacecraft body (see Fig. 5). The sensor A is sensitive to dust particles on inclined prograde orbits: observed from the spacecraft point of view, those particles come from the ecliptic south direction. Thus, we refer to the sensor A as the "south sensor". For similar reason, the sensor B is called the "ecliptic sensor" since its field of view fully scans the ecliptic during one spacecraft rotation. The field of view for both instruments is further constrained by the spacecraft structure, resulting in slightly different target area: $54.5 \mathrm{~cm}^{2}$ for the ecliptic sensor and $66.5 \mathrm{~cm}^{2}$ for the south sensor (see Sect. 2). As the ecliptic sensor looks into the Sun once per rotation, an additional metal-coated parylene foil of 0.3 micron thickness covers the instrument aperture. This foil prevents solar radiation from entering the sensor and heating it up beyond safe operations but it can be traversed by dust impactors. However, the sensitivity of the sensor is decreased as described in Sect. 3. In contrast, the south sensor has only a protection against the solar wind plasma, which does not decrease its sensitivity.

\section{Data description}

From 1974-353 to 1980-002 (in year and day-of-year format), the Helios dust experiment transmitted data of 235 dust impacts to the Earth. The impact identification and noise discrimination scheme is described in details by Grün (1981). For our analysis we used solely the following impact parameters: the date of the impact $t$, the distance to the Sun $r$, the true anomaly $\eta$, the azimuth angle $\theta$ and the ion impact charge $Q I$. In addition, the time-of-flight (TOF) mass spectra analyses by Grün (1981) were also taken into account.

The impactors detected with the south sensor and those obtained with the ecliptic sensor are shown in a $(\eta, \theta)$-diagram in Fig. 6, (Grün et al. 1980). The contour-lines represent the sensitive area of the instrument to populations on circular prograde orbits. Note that all possible values of the $\beta$ ratio $(0<\beta<1)$ for IDPs on prograde circular orbits have been taken into account for this calculation. This plot reveals a different distribution of the impactors in the $(\eta, \theta)$-space, depending on the detector. While the impactor distribution of the ecliptic sensor can almost be completely explained by impactors on circular or low eccentric orbits, highly eccentric orbits are needed to account for the impactors distribution of the south sensor. This fact was already discussed by Grün et al. (1980). According to the interpretation of this author, the ecliptic sensor was not sensitive to particles of low material density on high eccentric orbits because of the foil protecting the instrument against thermal radiation (see Sect. 2).

\section{Identification of ISD impactors}

In this section, we aim at finding criteria that allow us to discriminate ISD grains from the population of IDPs. Regarding the orbital elements of the IDP populations, we make the following assumptions:

a- The IDPs are all on bound prograde heliocentric trajectories.

b- The IDPs have low-inclined orbits $\left(<30^{\circ}\right)$.

c- The argument of perihelion $\omega$ is assumed to be equally distributed over $[0,2 \pi]$.

d- The longitude of ascending node $\Omega$ is assumed to be equally distributed over $[0,2 \pi]$.

The first assumption can be justified by the following fact: if one assumes the same dust number density, a spacecraft flying in the prograde direction should observe a much higher flux of particles on retrograde orbits than on prograde orbits. However, none of the performed in situ experiments detected a significant amount of retrograde dust particles, as reported in the analysis of the HEOS2 or Helios measurements (Hoffmann et al. 1975; Grün 1981). The three remaining assumptions describe the rough rotational symmetry of the zodiacal cloud (Fechtig 1989). On the other hand, the ISD particles are assumed to be injected into the heliosphere as described in Sect. 1 and are on 

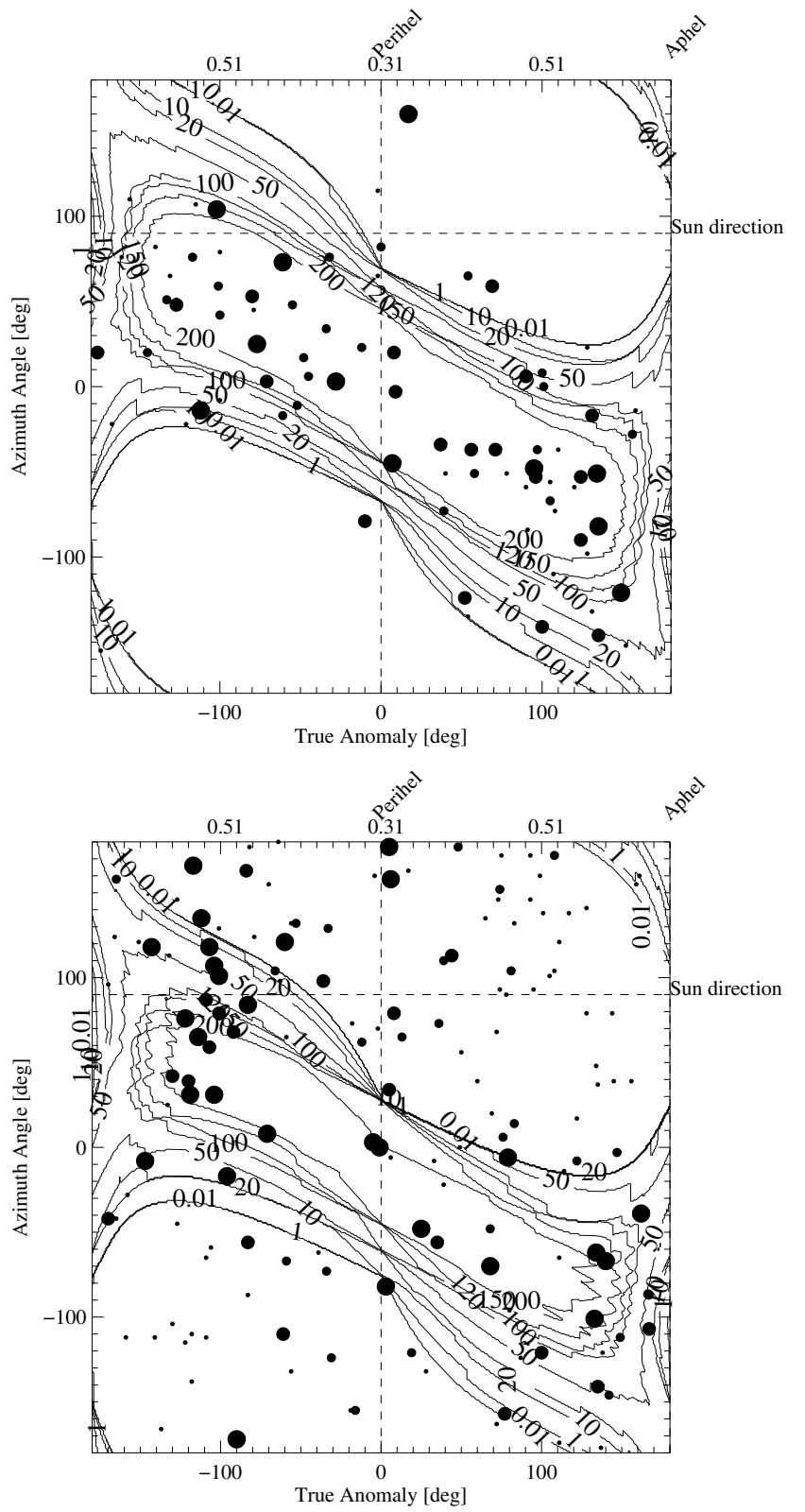

Fig. 6. Impactors detected by, respectively, the ecliptic sensor (on the top), and the south sensor (on the bottom) in a $(\eta, \theta)$-diagram. The impactors are represented by circles whose size is proportional to $Q I_{\mathrm{c}}$. The contour-lines give the sensitivity to interplanetary populations on circular orbits. The dotted vertical line and the horizontal lines give, respectively, the perihelion and the Sun direction. The upper axis indicates the heliocentric distance in AU.

hyperbolic trajectories. However, as mentioned in Sect. 2, we cannot simply rely on the impact velocities measured by the instrument to perform the discrimination between ISD and IDPs. Therefore, a carefully geometrical analysis, together with the impact ion charge measurement $Q I$ are required for a reliable identification of ISD grains.

The main goal of the geometrical analysis is identifying impactors in the data set presented in Fig. 6 the impactors that cannot be ISD grains. As mentioned in Sect. 1, ISD grains that are likely to be detected near the Sun (say less than $1 \mathrm{AU}$ ) must have a low charge-to-mass ratio, otherwise they will be deflected away by Lorentz interactions before they reach the inner solar system. Thus, gravitation and radiation pressure dominate for such grains, and their equation of motion is

$\ddot{\boldsymbol{r}}+\frac{G M_{\odot}(1-\beta)}{|\boldsymbol{r}|^{3}} \boldsymbol{r}=0$

where $M_{\odot}$ is the Sun's mass and $G$ the gravitational constant.

For a given impactor the spacecraft position in polar coordinates $\left(r_{\mathrm{sp}}, f_{\mathrm{sp}}\right)$ is derived from the angle $\eta$, and the $+X$ direction is defined by the velocity vector of the dust stream at infinity (injection velocity into the heliosphere). We can search then possible trajectories of ISD grains on collision course with the spacecraft. Solution hyperbola are parameterized by the $\beta$ value and the problem is equivalent to finding the impact parameter $b$ that ensures

$r_{\mathrm{sp}}=\frac{b^{2} v_{\infty}^{2}}{\tilde{\mu}}\left[1+\sqrt{\left(1+\frac{b^{2} v_{\infty}^{4}}{\tilde{\mu}^{2}}\right)} \cos \left(f_{\mathrm{sp}}-\frac{\delta}{2}+\frac{\pi}{2}\right)\right]^{-1}$,

where $\delta=\arcsin \left(\frac{1}{e}\right)$ is the deflection angle. For each hyperbola corresponding to a collision course, the detector area sensitive to the grain is found by calculating the theoretical impact velocity vector. Thus, impactors for which the sensitive area vanishes for all values of $\beta$ cannot have an interstellar origin and are discarded. As a result, Fig. 7 shows remaining potential ISD candidates. Note in particular that IDPs ejected almost radially from the Sun on hyperbolic orbit, also called $\beta$ particles (Wehry \& Mann 1999), cannot be confused with ISD grains. Although each of the remaining impactors in Fig. 7 may have an interstellar origin, they could also be accounted for particles on circular or low eccentric orbits, see Fig. 6. Therefore, a more detailed analysis, based on the impact charges, is necessary.

Figure 8 shows the impact charge generated by the impactors as function of the true anomaly angle $\eta$. A cluster of 7 impactors has been detected in a very narrow angular range $\left( \pm 1^{\circ}\right)$ around $\eta=135^{\circ}$ as shown by the vertical line. As those impacts have been detected over different orbits at almost exactly the same location, it is very unlikely that the grains have an interstellar origin. They may account for a periodic crossing of the spacecraft with big grains of a cometary trail, keeping the orbital elements of their parent body (owing to their size, such grains are less sensitive to radiation pressure). This scenario is supported by the relatively high impact charges of the cluster and should be verified in the future (in particular it could be interesting to know if those impacts match any known cometary trail). As a consequence, the impacts of this cluster are excluded from the following considerations.

The impact charges of the remaining impactors are asymmetrically distributed. Indeed, 12 particles with impact charges higher than $10^{-11} \mathrm{C}$ have been detected with $\eta<0$ while only 4 with $\eta>0$. As $\eta=0$ is the perihelion position, this means that an excess of high impact charges have been detected on the infalling leg of the orbit (the orbit segment moving into the ISD stream, see Fig. 2). We claim that this excess is not consistent with an interplanetary origin for the impactors. Indeed, if $v$ is the spacecraft velocity, it is obvious that $v(\eta)=v(-\eta)$. Thus, $n_{\text {imp }}$ being the number of impactors detected, this should lead to $n_{\mathrm{imp}}(\eta) \approx n_{\mathrm{imp}}(-\eta)$ in each impact charge range, owing to the 

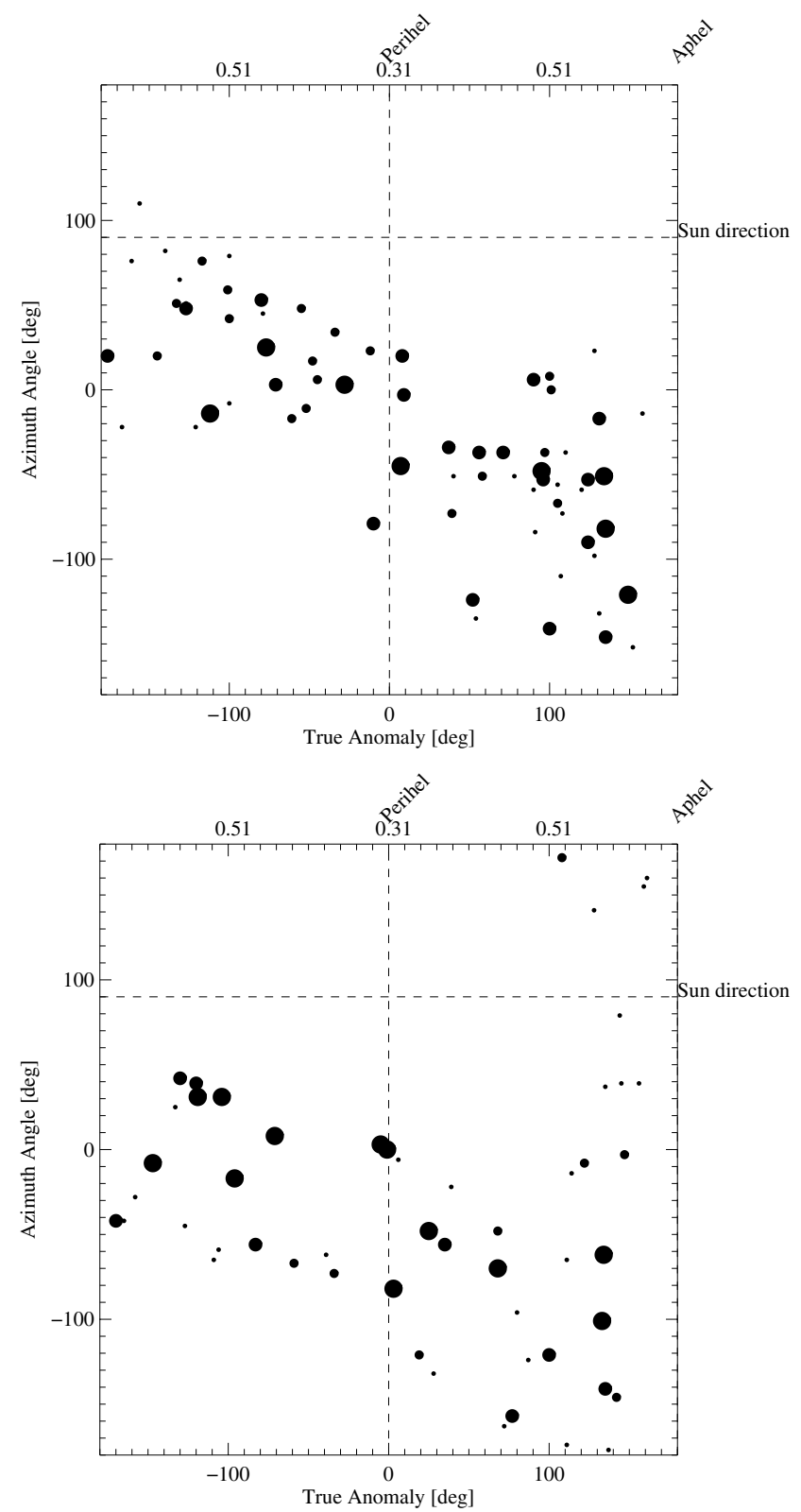

Fig. 7. Impactors detected by, respectively, the ecliptic sensor (on the top), and the south sensor (on the bottom) in a $(\eta, \theta)$-diagram. All impactors that are not compatible with an interstellar origin have been discarded, based on geometrical calculations. The dotted vertical line and the horizontal line gives, respectively, the perihelion and the Sun direction. The upper range indicates the heliocentric distance.

rough rotational symmetry of the zodiacal cloud. Furthermore, we will show that this asymmetric impact charge distribution can be explained by the contribution of an ISD population together with a population of IDPs on circular orbits.

Calculations were performed to find the theoretical impact velocity function $v_{\text {imp }}(\beta, \eta)$ of ISD grains on the instrument target. Then, using the calibration curve Eq. (1) of the instrument, the $\beta(m)$ ratio (see Fig. 1), and the equation of motion Eq. (3), we derived the theoretical impact charge yield function $Q_{\text {imp }}(\beta, \eta)$. Furthermore, we calculated the effective sensitive area $A_{\text {eff }}(\beta, \eta, \theta)$ of the instrument, both for ISD and IDPs on circular orbits. $A_{\text {eff }}$, defined as the target area exposed to

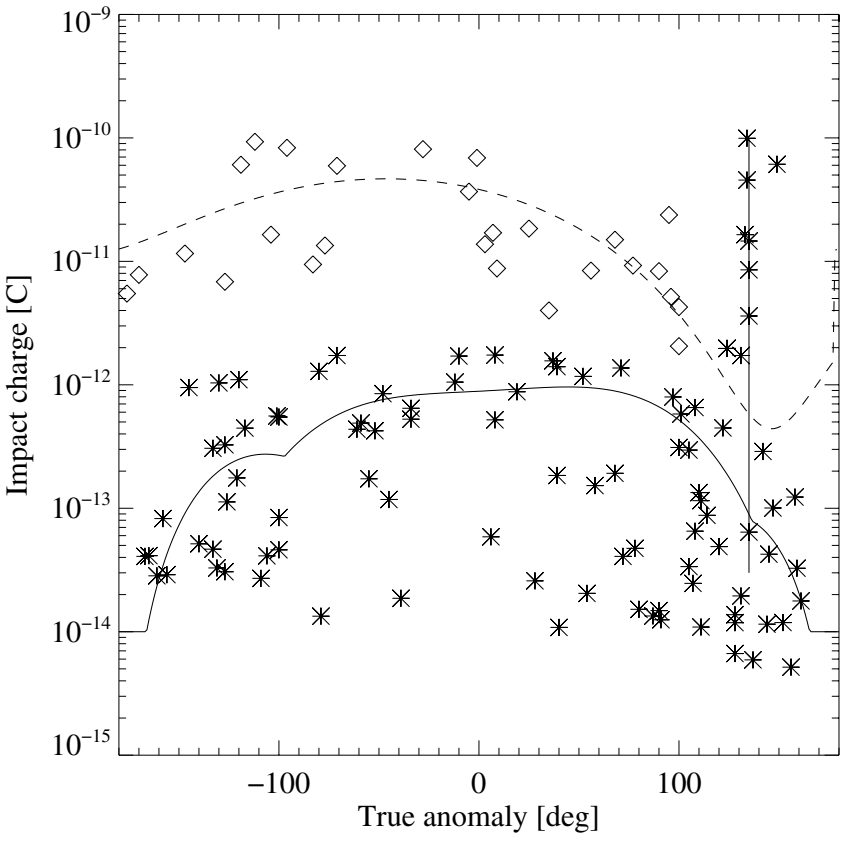

Fig. 8. Impact charges detected by the ecliptic and the south sensor. The solid line shows the mean impact charge expected for IDPs on circular orbits as function of $\eta$. The dashed line shows the theoretical impact charge generated by ISD grains for which $\beta=0.46$, the average value of $\beta$ found for the interstellar impactors. The vertical line shows a cluster of 7 impacts detected at almost exactly the same position of the spacecraft on its orbit, and may be due to a meteoroid stream intercepted by the spacecraft's orbit, see text.

particles entering the detector, is a function of the spacecraft position (given by $\eta$ ), of the instrument pointing (parameterized by $\theta$ ), and of the $\beta$ value of the grain. In addition, to take into account the relative motion of the spacecraft relative to the dust population, we define the effective sensitive area as the detector area required to register the same impact rate in the heliocentric inertial frame. As a consequence, the effective sensitivity for a dust population will be high for a pointing direction offering the largest target area to the impactors, combined with a high impact velocity. The theoretical impact velocities, impact charges and effective sensitive area for ISD and IDPs on circular orbits are plotted in Figs. 9-11 respectively. Note that in Fig. 11, the averaged value of $A_{\text {eff }}(\beta, \eta, \theta)$ over one spacecraft rotation is plotted.

The qualitative interpretation of the theoretical calculations leads to the following observations:

a- As shown in Fig. 11, the sensitivity for ISD grains increases from aphelion to perihelion, and reaches a maximum for $\eta \approx-30^{\circ}$. The position of the maximum does not depend on the value of $\beta$. The smaller the value of the $\beta$ ratio, the higher the maximum of the sensitive area, resulting in a higher sensitivity for big ISD grains. In contrast, on the right part of the orbit, from perihelion to aphelion, the sensitivity for ISDs decreases, reaching a minimum for $\eta \approx 140^{\circ}$. Thus, the probability function for ISD detection is not symmetric with respect to the line of apsides and the best geometrical conditions are met for $-180^{\circ}<\eta<90^{\circ}$. 

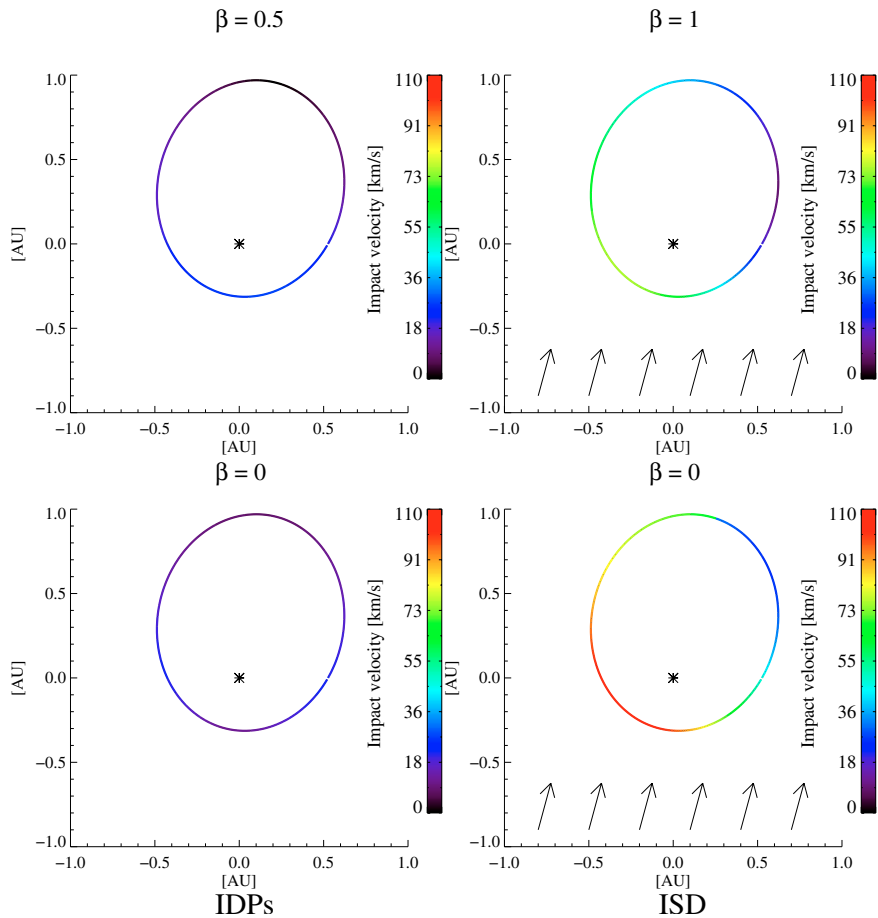

Fig. 9. Theoretical impact velocity values calculated for IDPs on circular orbits (on the left) and for ISD grains (on the right). For each population, two different $\beta$-ratios have been assumed for the calculations. The ISD stream direction for $\beta=1$ is symbolized by the arrows. Note the high impact speeds of ISD grains on the infall portion of the orbit, close to the perihelion.
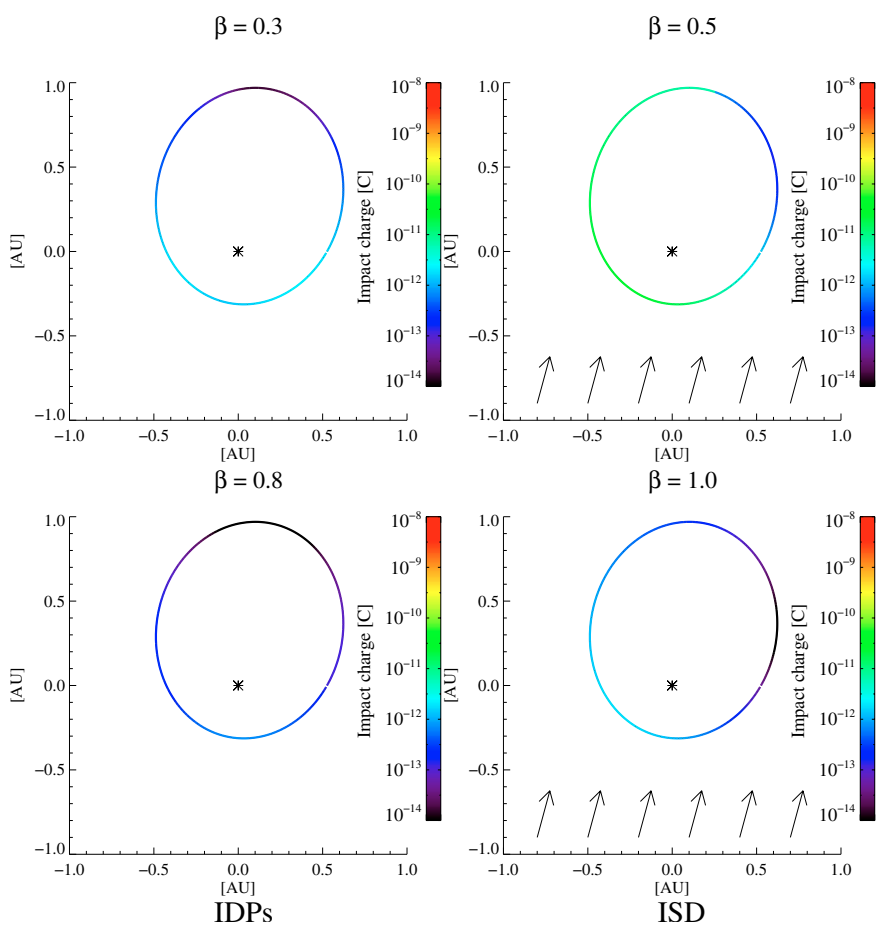

Fig. 10. Theoretical impact charge calculated for IDPs on circular orbits (on the left) and for ISD grains (on the right). For each population, two different $\beta$-ratio values have been assumed for the calculations. The ISD stream direction for $\beta=1$ is symbolized by the arrows. Note that big IDPs $(\beta \approx 0.3)$ can generate as much charges as small ISD grains $(\beta \approx 1)$. The highest impact charges, however, are generated by big ISDs, for spacecraft locations close to the aphelion.
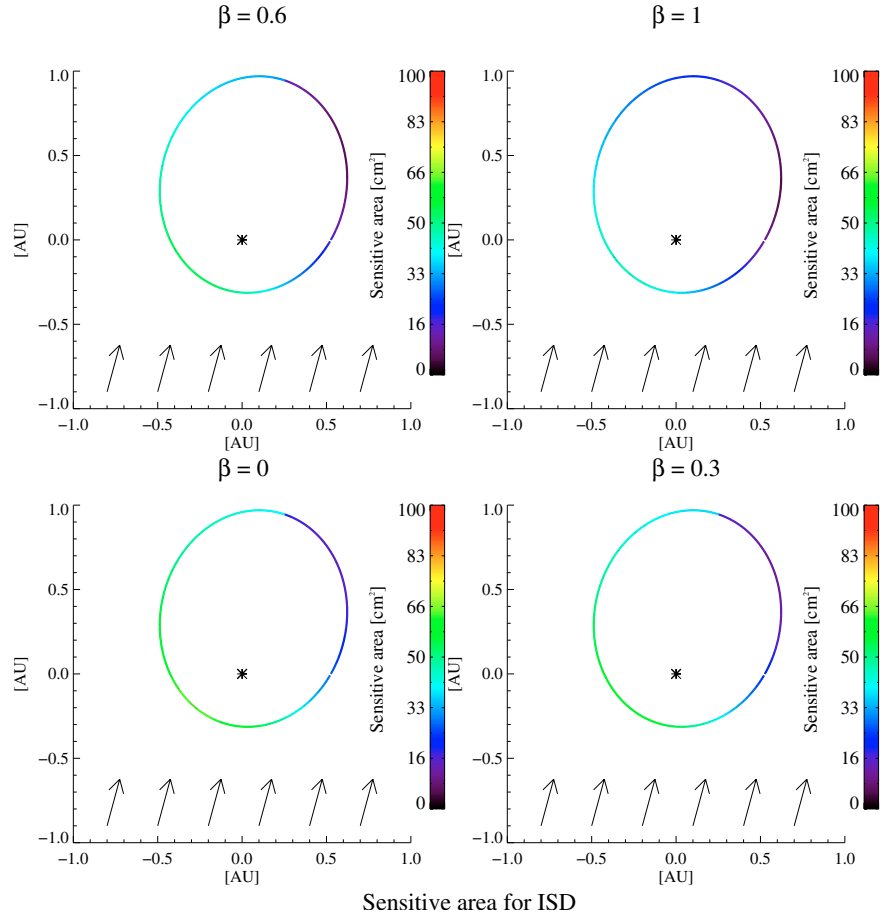

Fig. 11. Effective sensitive area for different populations of ISD grains, characterized by their $\beta$-ratio values. The sensitive area of both detectors have been taken into account and averaged over one spacecraft rotation.

b- Expected impact velocities for ISD particles are given in the right hand plots in Fig. 9 for two $\beta$-ratio values. Impact velocities up to $100 \mathrm{~km} \mathrm{~s}^{-1}$ are expected at $\eta \approx-40^{\circ}$ for ISD grains for which $\beta=0$. The maximum impact velocity is decreasing with increasing $\beta$-ratios as shown on the upper right hand plot. However, even for ISD with $\beta=1$, the impact velocity goes up to $60 \mathrm{~km} \mathrm{~s}^{-1}$. In contrast, the impact velocities expected for IDPs on circular orbits are much lower, as shown on the left hand plots. The maximum impact velocities are reached for $\eta \approx 30^{\circ}$. For big IDP grains $(\beta=0)$, the maximum impact velocity is about $30 \mathrm{~km} \mathrm{~s}^{-1}$ while for grains for which $\beta=0.5$ the maximum is less than $40 \mathrm{~km} \mathrm{~s}^{-1}$.

c- From the comparison of the expected ion charge for IDPs and ISD in Fig. 10, one can see significant differences in the shape of the $Q_{\text {imp }}(\beta, \eta)$. For the ISD grains, the maximum of the calculated impact charge is reached for $\eta \approx-40^{\circ}$ while for IDPs, the maximum is reached shortly after the perihelion. The difference between the impact charge generated by IDPs and those generated by ISD impactors still depends on the $\beta$ ratio assumed for each population. However, on the orbit part defined by $-180^{\circ}<\eta<90^{\circ}$, big ISD grains may generate much higher impact charges than IDPs, see Fig. 10.

These theoretical results were used to interpret the impact charges distribution in Fig. 8. The data can be well fitted using two dust populations: ISD grains, and IDPs on circular orbits, both characterized by their $\beta$-ratio. We can optimize the $\beta$-ratio for each population, in order to find the $Q_{\text {imp }}(\beta, \eta)$ functions that fit the data best as shown in Fig. 8. Impactors that 
generated high impact charges $\left(Q I>2 \times 10^{-12} \mathrm{C}\right)$ are best fitted by ISD grains for which $\beta \approx 0.4$, while the lower impact charge range is best fitted by IDPs on circular orbit with $\beta \approx 0.5$. The impact charge range values of ISD grains correspond to $\beta$-values ranging from 0.3 to 0.7 or a mass between $10^{-15} \mathrm{~kg}$ and $10^{-14} \mathrm{~kg}$ (see Fig. 1). Assuming a mass bulk density of about $2500 \mathrm{~kg} \mathrm{~m}^{-3}$ for silicate spheres, this mass range is equivalent to particle radii between 1 and $2 \mu \mathrm{m}$. However, the spread in the $\beta$ values around the best fit may also reflect difference in grains composition (see Fig. 15).

\section{Discussion}

Based on the argument developed in Sect. 4, we finally conclude that impactors described by $-180^{\circ}<\eta<90^{\circ}$ and $Q I>2 \times 10^{-12} \mathrm{C}$ are mainly from interstellar origin. About 27 impactors fulfill this criterion, with a standard deviation of about 5 impactors if one assume a Poisson distribution for the dust detection. As the orbital period of the Helios spacecraft was about 6 months, the orbit segment defined by $-180^{\circ}<\eta<90^{\circ}$ was flown 10 times between 1975 and 1980. If $t_{0}$ is the time when the spacecraft passed its aphelion and $t$ the time when the spacecraft reached the position $\eta=90^{\circ}$, $\Delta t=t-t_{0}$ can be calculated using the Kepler equation

$M=E-e \sin E$,

where $M$ is the mean anomaly, $e$ the eccentricity and $E$ the eccentric anomaly. Furthermore, $M$ is a function of time as

$M=\sqrt{\frac{\mu}{a^{3}}}\left(t-t_{0}\right)$,

where $a$ is the semi-major axis of the trajectory.

Since

$\tan \frac{\eta}{2}=\sqrt{\frac{1+e}{1-e}} \tan \frac{E}{2}$,

we derive $\Delta t$ and write the function $A_{\text {eff }}(\eta, \beta)$ as $A_{\text {eff }}(t(\eta), \beta)$. Time integration of $A_{\text {eff }}(t(\eta), \beta)$ for $\beta=0.4$ allows us to calculate the ISD flux value. Note that measurements were performed on average only $61.5 \%$ of the total time (Grün 1981). This factor must therefore be taken into consideration for the flux calculation. The resulting mean flux for $\beta=0.4$ is of $2.6 \pm 0.3 \times 10^{-6} \mathrm{~m}^{-2} \mathrm{~s}^{-1}$.

Interestingly, the $\beta$-ratio found for those ISD grains implies bigger sizes (about $1 \mu \mathrm{m}$ ) than the typical grains observed by Ulysses (radii about $0.3 \mu \mathrm{m}, \beta \approx 1.1$ (Landgraf et al. 2003)). In addition, the ISD flux measured is about 100 times smaller than the mean ISD flux measured between 3 AU and 5 AU. One reason may reside in the fact that the ISD stream component characterized by $\beta \approx 1$ could not be discriminated from the IDP background with the Helios sensors. Indeed, Fig. 8 shows that such ISD grains generate an impact charge around $10^{-12} \mathrm{C}$, that is in the same order as for IDPs. The ISD identification scheme is, therefore, less sensitive to small ISD grains, that may cause an underestimation of the total ISD flux. However, we show in the following that the ISD flux values measured with the Helios data are consistent with the general understanding of the ISD stream alteration in the heliosphere.
Analysis of the Ulysses dust data set showed that the differential mass distribution of ISD grains has a negative slope for grain size bigger than about $0.3 \mu \mathrm{m}$ (Landgraf et al. 2000). Therefore, a question arises from our results: why are the big ISD grains dominant in the Helios data sample? We can show qualitatively and quantitatively that the combined effects of gravitation focusing and radiation pressure filtering result in a strongly modified mass distribution of the ISD close to the Sun.

The dynamics of particles characterized by small $\beta$ ratios is dominated by gravitation forces. The gravitation focusing effect induces on such particles an enhancement of the heliocentric flux close to the Sun, and in particular behind the Sun with respect to the ISD stream downstream direction (Fahr 1968). Two collaborating effects are responsible for this flux enhancement. First, the heliocentric velocity of particles close to their perihelion on a hyperbolic orbit is higher than their injection velocity. Second, the gravitation focusing is responsible for an enhancement of the dust number spatial density in the vicinity of the Sun. Because its orbit is relatively close to the Sun, the Helios spacecraft benefits from both effects and the ISD flux is enhanced by a factor given by:

$\frac{F}{F_{\infty}}=\frac{v}{v_{\infty}} \frac{n}{n_{\infty}}$

where $F_{\infty}$ is the ISD flux in the heliocentric system at the heliosphere boundaries, $F$ is the flux value in the spacecraft frame at the spacecraft location, $v_{\infty}$ is the ISD injection velocity, $v$ is the velocity of the grains relative to the spacecraft, $n_{\infty}$ is the dust spatial density at the heliosphere boundaries, and $n$ is the dust spatial density at the spacecraft location. Note that, during orbit sections where the Helios spacecraft is flying toward the ISD stream, the dust flux is further enhanced by the higher velocity $v$. The flux enhancement factor has been calculated and plotted in Fig. 12. The curve is not symmetric with respect to the perihelion $\left(\eta=0^{\circ}\right)$ since calculations have been made in the spacecraft reference frame. As shown in this plot, an enhancement factor up to 6 can be expected around the spacecraft perihelion for particles with small $\beta$ values.

A second physical mechanism explains the dominant contribution of big ISD impactors in the Helios data set. As seen in Fig. 13, the Helios orbit lies inside the exclusion zones shaped by the solar radiation pressure. In particular, typical grains with $\beta=1.1$ as seen by Ulysses, are prevented by the radiation pressure from reaching the Helios spacecraft. As a consequence, only these ISD grains with $\beta<1$ can be detected. Note, however, that the detection of smaller grains $(\beta=1.1)$ was theoretically possible on a very small orbit segment around the perihelion, when the spacecraft crossed the boundary for $\beta=1.1$. (see Fig. 13). As the ISD identification scheme is less sensitive to such grain sizes, this contribution may not have been identified.

The advantage of 5 years integration time is to compensate for the small spatial density of big ISD grains. One should also notice that the south sensor is mostly sensitive to big ISD grains. Indeed, the trajectories of those grains form a revolution hyperboloid with the Sun as focal point. Thus, particles injected below the ecliptic plane will be deviated toward the north, and the smaller the $\beta$-ratio, the stronger the deviation. Big grains are therefore better detected by the south sensor than 

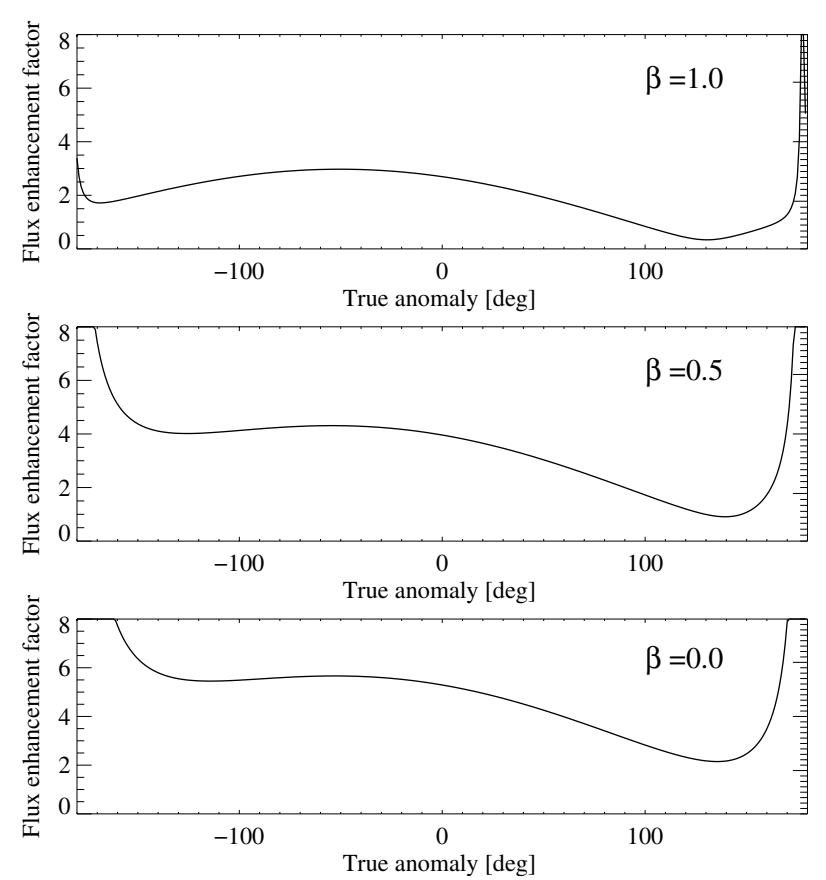

Fig. 12. ISD flux enhancement factor due to gravitational focusing as function of the true anomaly, calculated in the spacecraft reference frame. Three $\beta$-ratios have been considered. The gravitational focusing effect is stronger for big particles.

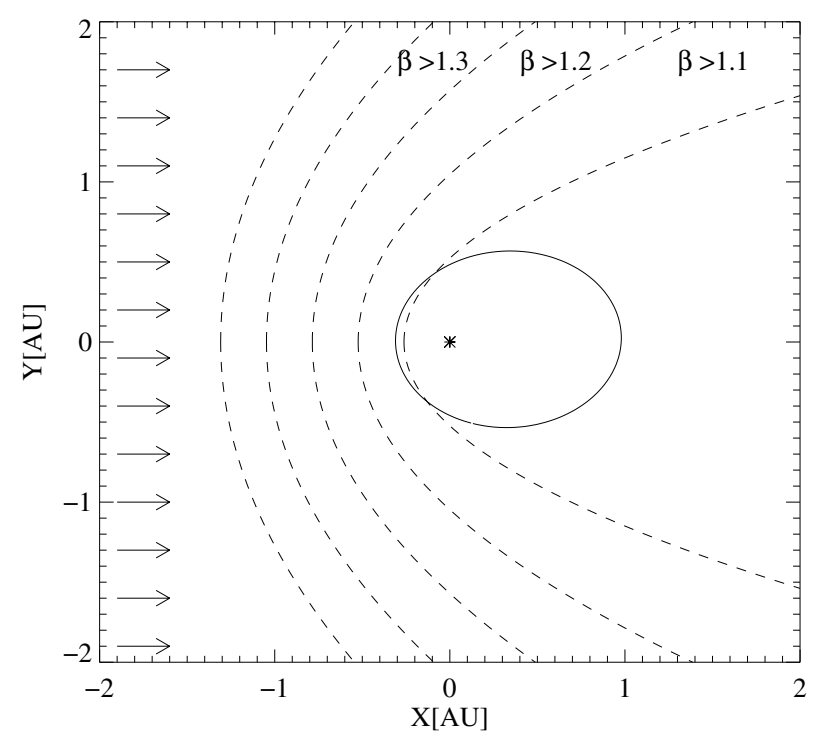

Fig. 13. Trajectory of the Helios spacecraft. The $X$-axis indicates the injection velocity of the ISD stream. The dashed lines are the boundaries of the exclusion zones corresponding to different $\beta$ values.

ISD grains of smaller masses, for which the field-of-view vanishes.

From the micron-size grains flux calculated on the Helios trajectory, one can infer the spatial mass density for those grains at the Heliopause. This calculation simply needs a correction to the flux value found by the flux enhancement factor. This leads to a flux value for micron-size grains $(\beta \approx 0.4)$ of around $0.65 \times 10^{-6} \mathrm{~m}^{2} \mathrm{~s}^{-1}$, equivalent to a spatial mass density of about $3 \pm 3 \times 10^{-25} \mathrm{~kg} \mathrm{~m}^{-3}$. The spatial mass density value derived from the Ulysses measurements for micron-size
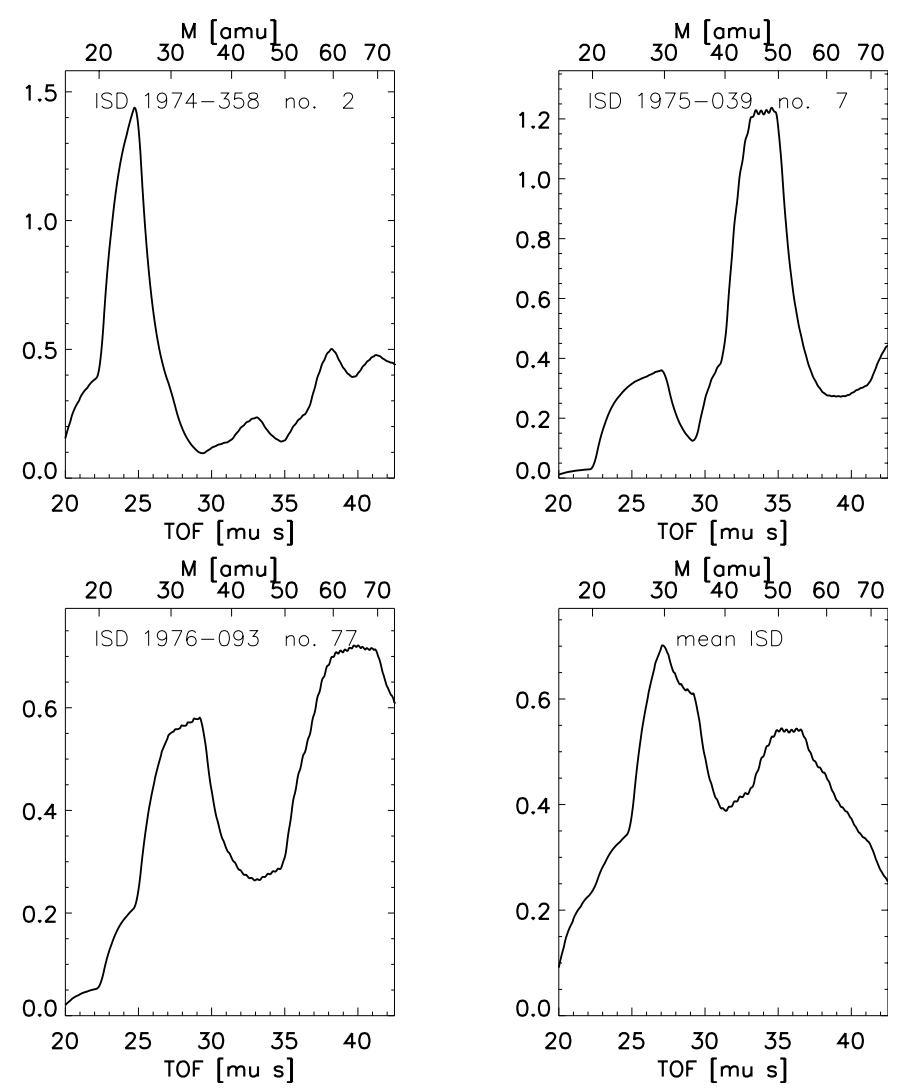

Fig. 14. Spectra of interstellar dust grains recorded by Helios. Upper left: chondritic types (Ch); upper right: Fe-type spectrum; lower left: high mass spectrum; lower right: mean spectrum of all ISD grains identified. All spectra have been normalized to constant area.

grains is about $1 \pm 3 \times 10^{-24} \mathrm{~kg} \mathrm{~m}^{-3}$, (Landgraf et al. 2000). Although those two values are comparable, additional effects must be responsible for the lower value found by Helios. While sublimation is not expected to play an important role outside 0.3 AU for dust grains (Krivov et al. 1998), sputtering by solar wind may shift the grain size distribution toward smaller sizes (Mukai \& Schwehm 1981). However, numerical applications show that this effect can not be responsible for the difference in spatial density of big grains found in the Helios data and Ulysses data. Indeed, ISD grains need about 6 months, starting at around $4 \mathrm{AU}$ (mean heliocentric distance for detection of ISD by Ulysses ) to reach $0.3 \mathrm{AU}$. This exposure time to the solar wind particles is much too low for an erosion rate value of about $1 \times 10^{-16} \mathrm{~g} \mathrm{~cm}^{-2} \mathrm{~s}^{-1}$ (Mukai \& Schwehm 1981) to explain a significant shift of the ISD grain size distribution. Thus, the relatively small discrepancy between the Helios and Ulysses data for inferred spatial mass density remains unexplained and will be investigated in the future.

The analysis of the time-of-flight spectra provides important compositional clues on the big ISD grains. Spectral data of the 235 impacts recorded during the Helios mission were stored in a tables of 12 values corresponding to mass intervals of 5 a.m.u. width from 16 to 75 a.m.u. These values were folded with a reference peak for Helios spectra (Braun 1977) and the spectra were reconstructed. Figure 14 shows examples of three extreme cases of ISD spectra and the mean spectrum of all 


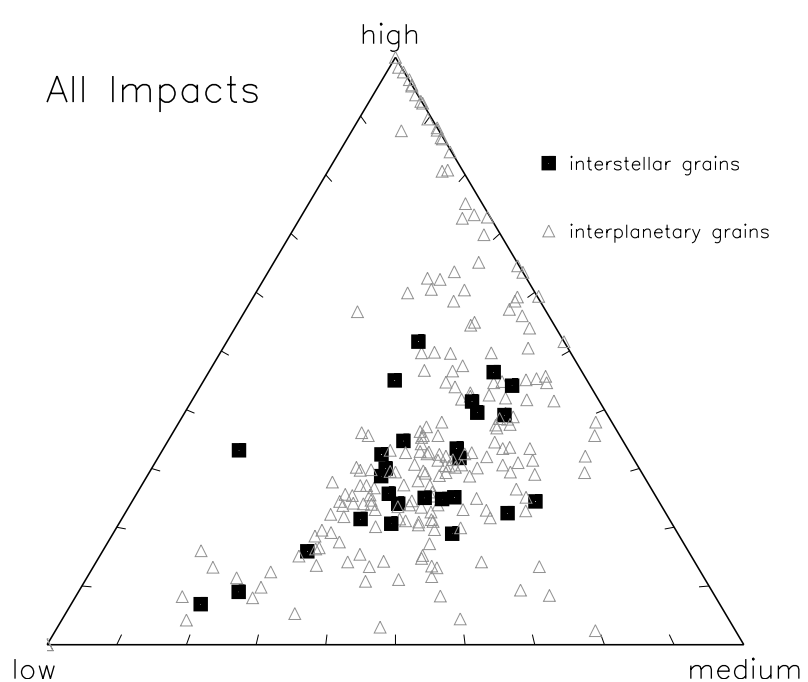

Fig. 15. Three-phase diagram (cf. Fig. 4) of interplanetary and interstellar dust spectra recorded by Helios.

interstellar particles. Masses around 30 a.m.u. compatible with silicates dominate the spectrum. A second peak of iron and/or molecular ions is visible between 50 and 60 a.m.u. The wide variety of compositions of spectra recorded by the Helios instrument in interplanetary space is displayed in Fig. 15. High ion mass end-member compositions containing mostly ions of masses above 50 a.m.u. are visible. Interstellar grains cover the region of low ion masses to moderate amounts of medium and high ion masses is seen. No clustering of single minerals is observed but rather a continuous transition from the predominance of low to high ion masses, indicating that individual grains are a varying mixture of various minerals and carbonaceous compounds.

Acknowledgements. This work was supported by the Max Planck Institut for Nuclear Physics, Heidelberg, Germany

\section{References}

Altobelli, N., Kempf, S., Landgraf, M., et al. 2003, J. Geophys. Res. Altobelli, N., Kempf, S., Krüger, H., Landgraf, M., \& Grün, E. 2004, J. Geophys. Res., in press

Baguhl, M., Hamilton, D. P., Grün, E., et al. 1995, Science, 268, 1016
Braun, G. 1977, Untersuchung der Eigenschaften der HeliosStaubsensoren mit Hilfe von Simulationsexperimenten, Diploma Thesis Universität Heidelberg

Brownlee, D. E. 1978, Cosmic Dust, ed. J. McDonnell (Wiley, Chichester)

Burns, J. A., Lamy, P. L., \& Soter, S. 1979, Icarus, 40, 1

Dalmann, B., Grün, E., \& Kissel, J. 1977, Planet. Space Sci., 25, 135

Dietzel, H., Eichhorn, G., Fechtig, H., et al. 1973, J. Phys. E Scientific Instruments, 6, 209

Draine, B. T., \& Lee, H. M. 1984, ApJ, 285, 89

Eichhorn, G. 1978a, Planet. Space Sci., 26, 463

Eichhorn, G. 1978b, Planet. Space Sci., 26, 469

Fahr, H. J. 1968, Astrophys. Space Sci., 2, 474

Fechtig, H. 1989, Z. Naturforsch., 44a, 877

Frisch, P. C., Dorschner, J. M., Geiss, J., et al. 1998, BAAS, 31, 661

Grün, E. 1981, Physikalische und chemische Eigenschaften des interplanetaren Staubesmessungen des Mikrometeoritenexperimentes auf Helios (Bundesministerium für Forschung und Technologie, Forschungsbericht W 81-034)

Grün, E., Pailer, N., Fechtig, H., \& Kissel, J. 1980, Planet. Space Sci., 28,333

Grün, E., Fechtig, H., Giese, R. H., et al. 1992a, A\&AS, 92, 411

Grün, E., Fechtig, H., Hanner, M., et al. 1992b, Space Sci. Rev., 60, 317

Grün, E., Gustafson, B., Mann, I., et al. 1994, A\&A, 286, 915

Gustafson, B. 1994, Ann. Rev. Earth Planet. Sci., 22, 553

Gustafson, B. S., \& Misconi, N. 1979, Nature, 282, 276

Hoffmann, H.-J., Fechtig, H., Grün, E., \& Kissel, J. 1975, Planet. Space Sci., 23, 215

Kimura, H., \& Mann, I. 1998, ApJ, 499, 454

Krivov, A., Kimura, H., \& Mann, I. 1998, Icarus, 134, 311

Landgraf, M., Baggaley, W. J., Grün, E., Krüger, H., \& Linkert, G. 2000, J. Geophys. Res., 105, 10343

Landgraf, M., Krüger, H., Altobelli, N., \& Grün, E. 2003, J. Geophys. Res., 108, 5

Levy, E., \& Jokipii, J. 1976, Nature, 264, 423

Li, A., \& Greenberg, J. M. 1997, A\&A, 323, 566

Mann, I., \& Grün, E. 1996, Adv. Space Res., 17, 99

Mathis, J. S., Rumpl, W., \& Nordsieck, K. H. 1977, ApJ, 217, 425

Morfill, G., \& Grün, E. 1979, Planet. Space Sci., 27, 1283

Mukai, T., \& Schwehm, G. 1981, A\&A, 95, 373

Sparrow, R. 1977, in 25th Annual Conf. on Mass Spectrometry and Allied Topics, Washington D.C.

Srama, R., Bradley, J., Grün, E., et al. 2003, SSR, in press

Wehry, A., \& Mann, I. 1999, A\&A, 341, 296

Wiley, W., \& McLaren, I. 1955, Rev. Sci. Instr., 26, 1150

Witte, M., Rosenbauer, H., Banaszkiewicz, M., \& Fahr, H. 1993, Adv. Space Res., 13, 121 